\title{
Control Laws for a Dual-Spin Stabilized Platform
}

\author{
K. B. Lim * and D. D. Moerder ${ }^{\dagger}$ \\ NASA Langley Research Center, Hampton, Virginia, 23681, USA
}

\begin{abstract}
This paper describes two attitude control laws suitable for atmospheric flight vehicles with a steady angular momentum bias in the vehicle yaw axis. This bias is assumed to be provided by an internal flywheel, and is introduced to enhance roll and pitch stiffness. The first control law is based on Lyapunov stability theory, and stability proofs are given. The second control law, which assumes that the angular momentum bias is large, is based on a classical PID control. It is shown that the large yaw-axis bias requires that the PI feedback component on the roll and pitch angle errors be cross-fed. Both control laws are applied to a vehicle simulation in the presence of disturbances for several values of yaw-axis angular momentum bias. It is seen that both control laws provide a significant improvement in attitude performance when the bias is sufficiently large, but the nonlinear control law is also able to provide improved performance for a small value of bias. This is important because the smaller bias corresponds to a smaller requirement for mass to be dedicated to the flywheel.
\end{abstract}

\section{Introduction}

This paper describes and demonstrates a Lyapunov-based nonlinear control law for an atmospheric flight vehicle that is "dual-spin" stabilized - that is, it includes an angular momentum bias along its yaw axis to gyrically stiffen its roll-pitch attitude dynamics. It has been observed ${ }^{1}$ that, if the bias momentum is sufficiently large, the vehicle's roll-pitch dynamics approach those of an undamped second-order linear oscillator whose natural frequency scales with the magnitude of the bias.

If this bias is sufficiently large - i.e. the system becomes gyrically dominant - the magnitude and bandwidth of control activity necessary to maintain a commanded attitude against disturbances can be dramatically reduced $;{ }^{1}$ furthermore, the approximately linear dynamics associated with gyric dominance are easily stabilized with a linear control law.

Although a large yaw-axis angular momentum bias is desirable for pitch-roll stability and disturbance rejection, the fact that it is, in all probability, produced by a dedicated flywheel means that having a large bias tends to increase the vehicle's mass.

Designing a dual-spin atmospheric flight vehicle requires effectively trading the mass penalty associated with the flywheel against the advantages of being able to choose slower, less powerful fluidic control effectors for stabilization and command tracking. These advantages can be mundanely enhancing, e.g. cheaper or lighter control effectors, or they can potentially enable adoption of an emerging control effector technology such as many current morphing technologies - that have difficulty in matching the authority or bandwidth available in traditional hinged control surfaces.

The control issue that arises in selecting the "smallest possible" bias momentum flywheel for a given dualspin-stabilized vehicle is that the gyric coupling in the dynamics is quadratic in its attitude rates, with this

*Senior Aerospace Technologist, Dynamic Systems \& Controls Branch, Kyong.B.Lim@nasa.gov

${ }^{\dagger}$ Senior Aerospace Technologist, Dynamic Systems \& Controls Branch, Daniel.D.Moerder@nasa.gov

$$
1 \text { of } 19
$$

American Institute of Aeronautics and Astronautics 
nonlinearity only becoming insignificant if the bias momentum becomes dominatingly large; or alternatively, if it can be guaranteed that the vehicle's attitude rates will be negligible, even in the face of disturbances. The consequence of this is that, for modest levels of angular momentum bias, a nonlinear flight control law is required.

In the sequel, a Lyapunov-based nonlinear attitude control law for a rigid vehicle carrying a constant angular momentum bias is presented, and global stability proofs are provided for the case of time-varying commanded attitude, and for special cases in which the commanded attitude is constant, or in which there is feedback only of the attitude rates. These latter two simplifications provide insights that lead to a simple approach for selecting the gains.

For comparison, a classical linear controller structure is described for the case where the vehicle is gyrically dominant (approximately linearizing the dynamics) with its angular momentum bias aligned with the yaw axis. Both controller structures are applied to the thrust-levitated research vehicle described in [2]. While undergoing substantial aerodynamic disturbance, the vehicle is controlled to execute a maneuver in which it rises vertically, then transitions to horizontal flight. The maneuver is simulated for identical disturbances with both linear and Lyapunov controllers, for several magnitudes of angular momentum bias. It is seen that the Lyapunov controller provides good performance with a very small angular momentum bias - corresponding to a flywheel system of no more than one percent of the vehicle mass - while the linear controller requires a dominatingly large angular momentum bias to provide comparable attitude performance

- corresponding to a flywheel comprising up to five percent of the vehicle mass.

The next section presents the Lyapunov-based controller and the vehicle dynamics for which it is intended. The stability theorems are given, along with a simple approach for selecting gains. Proofs of the theorems are given in Appendix A. Section 3 describes a classical controller for the gyrically dominant case. Section 4 describes the particular dynamics of the simulated vehicle and disturbance environment for which the controllers are compared, and presents the results. Section 5 presents conclusions.

\section{Attitude Control via Lyapunov Approach}

The control laws in this study are intended for rigid body systems with dynamics that include a constant angular momentum bias, denoted by the symbol $h_{B}$ :

$$
\begin{gathered}
\dot{v}=-\omega^{\times} v+C_{b o} g_{o}+\frac{1}{m} \phi_{f}+\dot{v}_{c}-\frac{1}{m} \dot{p}_{s} \\
J \dot{\omega}=-\omega^{\times}\left(J \omega+h_{B}\right)+\phi_{\tau}+\tau_{c}-\omega^{\times} h_{s}-\dot{h}_{s}
\end{gathered}
$$

where

$$
\begin{gathered}
\dot{v}_{c}=\frac{1}{m}\left[\dot{c}^{\times} \omega+c^{\times} \dot{\omega}+\omega^{\times} c^{\times} \omega\right] \\
\tau_{c}=c^{\times} C_{b o} g_{o}-\dot{c}^{\times} v-c^{\times} \dot{v}-\dot{J} \omega
\end{gathered}
$$

Equations (1,2) are given in body coordinates, and $v$ is translational velocity, $m$ is mass, $c$ is the vector from the body axes origin to the center of mass, $\omega$ is vehicle angular velocity, $J$ is the overall moment of inertia, and $C_{b o} g_{o}$ is the gravitational acceleration vector transformed to body axes from NED coordinates. The control laws developed in the sequel assume that the angular momentum bias $h_{B}$ is assumed to be aligned with the $z$-body axis. The symbols $\phi_{f}$ and $\phi_{\tau}$ denote control forces and torques. In $(3,4), \tau_{c}$ and $\dot{v}_{c}$ are secondary torques and forces, mostly associated with off-CG effects, where $c$ is the vector from origin to center of mass. The quantities $p_{s}$ and $h_{s}$ are internal linear and angular momenta associated with moving 
masses inside the vehicle. These generally would include momenta associated with rotating masses in the propulsion system, proof-mass dampers, and other such components.

The first and most general Lyapunov controller in this section causes the vehicle to follow a commanded attitude history $\bar{\nu}(t)$ that corresponds to a angular rate command $\bar{\omega}(t)$. These are related by the quaternion expression

$$
\dot{\bar{\nu}}=\frac{1}{2}[\bar{\nu}]\left(\begin{array}{c}
0 \\
\bar{\omega}
\end{array}\right) ; \quad \bar{\nu}^{T} \bar{\nu}=1
$$

where

$$
[\bar{\nu}] \triangleq\left[\begin{array}{cccc}
\bar{\nu}_{0} & -\bar{\nu}_{1} & -\bar{\nu}_{2} & -\bar{\nu}_{3} \\
\bar{\nu}_{1} & \bar{\nu}_{0} & -\bar{\nu}_{3} & \bar{\nu}_{2} \\
\bar{\nu}_{2} & \bar{\nu}_{3} & \bar{\nu}_{0} & -\bar{\nu}_{1} \\
\bar{\nu}_{3} & -\bar{\nu}_{2} & \bar{\nu}_{1} & \bar{\nu}_{0}
\end{array}\right] \triangleq[\bar{\nu}, \quad \bar{B}(\bar{\nu})], \quad \bar{B} \in \mathcal{R}^{4 \times 3}
$$

Denoting the actual vehicle attitude as $\nu$, the tracking errors are

$$
\begin{aligned}
& \delta \omega \triangleq \omega-\bar{\omega} \\
& \delta \nu \triangleq \nu-\bar{\nu}
\end{aligned}
$$

where $\delta \nu$ and $\delta \omega$ can be shown to satisfy

$$
\delta \dot{\nu}=\frac{1}{2}[\bar{\nu}]\left(\begin{array}{c}
0 \\
\delta \omega
\end{array}\right)+\frac{1}{2}[\omega] \delta \nu
$$

where

$$
[\omega] \triangleq\left[\begin{array}{rrrr}
0 & -\omega_{1} & -\omega_{2} & -\omega_{3} \\
\omega_{1} & 0 & \omega_{3} & -\omega_{2} \\
\omega_{2} & -\omega_{3} & 0 & \omega_{1} \\
\omega_{3} & \omega_{2} & -\omega_{1} & 0
\end{array}\right]
$$

Theorem 1 (Arbitrary Commanded Attitude) For the rotational equations of motion as given in (2), the feedback control torques

$$
\phi_{\tau}=\phi_{g y r o}+G_{\omega}(\omega-\bar{\omega})+G_{\nu}(\nu-\bar{\nu})+J \dot{\bar{\omega}}-\tau_{c}
$$

guarantees that the tracking excursions relative to the commanded set of attitude and angular rates, $\bar{\nu}$ and $\bar{\omega}$, will be globally asymptotically stable if the gains satisfy the following conditions:

$$
\begin{aligned}
& G_{\nu}=-\frac{1}{2} k_{\nu} J^{-1} \bar{B}^{T}, \quad k_{\nu} \in \mathcal{R}, \quad k_{\nu}>0, \\
& G_{\omega}=P J, \quad P \in \mathcal{R}^{3 \times 3}, \quad P<0
\end{aligned}
$$

$$
3 \text { of } 19
$$


where

$$
\phi_{\text {gyro }}=\omega^{\times}\left(J \omega+h_{B}\right)
$$

Noting that the inherent open-loop net gyroscopic torque on the vehicle is $-\omega^{\times}\left(J \omega+h_{B}\right)$ as given in equation (2), the feedback torque, $\phi_{\text {gyro }}$, as given in equation (14) is a feedback linearization term. The proofs of Theorem 1 and subsequent Theorems 2 and 3 are outlined in the Appendix. Global asymptotic stablility means that $\delta \omega \rightarrow 0_{3 \times 1}$, and $\delta \nu \rightarrow 0_{4 \times 1}$, as $t \rightarrow \infty$, for any initial condition dispersion about $\bar{\omega}$ and $\bar{\nu}$.

The next theorem restricts the control problem to tracking a fixed, rather than moving, commanded attitude. This permits the introduction of two tuning parameters: $\rho$ and $\epsilon$ in the $\phi_{\text {gyro }}$ expression from equation (14). For Theorem 2,

$$
\phi_{\text {gyro }}(\epsilon, \rho)=\epsilon \omega^{\times} J \omega+\rho \omega^{\times} h_{B}
$$

where

$$
\begin{aligned}
& \rho \in \mathcal{R}: 0 \leq \rho \leq 1 \\
& \epsilon \in \mathcal{R}: 0 \leq \epsilon \leq 1
\end{aligned}
$$

This permits the designer to separately scale the degree to which the control law is devoted to feedback linearization, via $\epsilon$, or to feeding back the gyroscopic torques associated with the angular momentum bias, via $\rho$. Note also that the now-constant $\bar{\nu}$ gives constant $\bar{B}$ which, in equation (12), results in constant $G_{\nu}$.

Theorem 2 (Fixed Commanded Attitude) For the rotational equations of motion as given in (2), and the scaled open-loop gyroscopic torque expression (15), the feedback control torques

$$
\phi_{\tau}=\phi_{g y r o}(\epsilon, \rho)+G_{\omega} \omega+G_{\nu}(\nu-\bar{\nu})-\tau_{c}
$$

guarantee that the excursions relative to the commanded set of attitude, $\bar{\nu}$, will be globally asymptotically stable if the gains satisfy the following conditions:

$$
\begin{aligned}
G_{\nu}= & -\frac{1}{2} k_{\nu} J^{-1} \bar{B}^{T}, \quad k_{\nu} \in \mathcal{R}, \quad k_{\nu}>0 \\
G_{\omega} & =\left[P-(1-\rho) \Delta_{\lambda}\right] J, \quad P \in \mathcal{R}^{3 \times 3}, \quad P<0 \\
\Delta_{\lambda} & =\delta_{\lambda}\left[\begin{array}{ccc}
0 & 1 & 0 \\
1 & 0 & 0 \\
0 & 0 & 0
\end{array}\right], \quad \delta_{\lambda} \triangleq \frac{1}{2}\left(\lambda_{p_{1}}-\lambda_{p_{2}}\right)
\end{aligned}
$$

where $\phi_{\text {gyro }}, \rho$, and $\epsilon$ are given by equations $(15,16,17)$, and $\lambda_{p_{1}}$ and $\lambda_{p_{2}}$ are

$$
\lambda_{p_{i}} \triangleq \frac{h_{B}}{J_{i}}, \quad i=1,2
$$

The $\lambda_{p_{i}}$ of the above Theorem are interpreted by recalling ${ }^{2}$ that $\sqrt{\lambda_{p_{1}} \lambda_{p_{2}}}$ is the undamped natural frequency of the linear roll-pitch dynamics that occur when the system becomes gyrically dominant; that is, when $\|J \omega\| /\left\|h_{B}\right\| / \rightarrow 0$. 
As a control problem, Theorem 2 is a subset of Theorem 1. However, the restrictive set of control problems addressed in Theorem 2 allows a more flexible feedback control structure in terms of incorporating the scaling parameter for feedback-linearization $\epsilon$ and the scaling factor $\rho$ for feeding back the bias momentum induced gyroscopic torque. The control law in Theorem 2 guarantees that the vehicle attitude excursions relative to a constant commanded attitude, $\bar{\nu}$, will be globally asymptotically stable.

From Theorem 2, the net angular velocity feedback components in equation (18) can be expressed as follows:

$$
\begin{aligned}
\phi_{\tau}(\omega) & =\phi_{\text {gyro }}+G_{\omega} \omega \\
& =\left[P+\epsilon \omega^{\times}-Q(\rho)\right] J \omega
\end{aligned}
$$

where

$$
Q(\rho) \triangleq \rho\left[\begin{array}{ccc}
0 & -\lambda_{p_{2}} & 0 \\
\lambda_{p_{1}} & 0 & 0 \\
0 & 0 & 0
\end{array}\right]+(1-\rho) \Delta_{\lambda}
$$

We make the following observations:

- The gain, $Q(\rho)$, is a convex combination of a matrix resembling the state-space matrix of an undamped, roll-pitch-gyrically dominated system, and the sparse symmetric matrix, $\Delta_{\lambda}$ given by equation (21). If $h_{B}=0$, i.e., in the absence of bias angular momentum, $Q(\rho)=0_{3 \times 3}$.

- If $J_{1}=J_{2}$, i.e., the vehicle is inertially axi-symmetric about its yaw axis,

$$
\lambda_{1}=\lambda_{2}=\lambda_{o} \quad \Rightarrow \quad \Delta_{\lambda}=0_{3 \times 3} \quad \text { and } \quad Q(\rho)=\rho\left(\begin{array}{c}
0 \\
0 \\
\lambda_{o}
\end{array}\right)^{\times}
$$

The design choice $(\rho=0, \epsilon=0)$ will require no feedback torque proportional to both the gyroscopic torque due to the presence of bias angular momentum nor the inherent gyroscopic torque induced by vehicle angular velocities. This means that for a vehicle with bias angular momentum and which is inertially axi-symmetric about the yaw axis, a constant gain angular velocity feedback,

$$
\phi_{\tau}(\omega)=P J \omega, \quad P<0
$$

will ensure asymptotic stability (along with appropriate attitude error feedback).

- The governing equation for the closed loop attitude response in Theorem 2 is

$$
J \delta \dot{\omega}=-(1-\epsilon) \omega^{\times} J \omega-(1-\rho) \omega^{\times} h_{B}+G_{\omega} \delta \omega+G_{\nu} \delta \nu
$$

The ubiquitous kinematic nonlinearity is evident. On the other hand, only the value of the design parameter, $\epsilon$, affects the presence of nonlinear dynamics in the closed loop response. In particular, only the value of $\epsilon=1$ ensures that there is an exact feedback linearization of the inherent nonlinear gyroscopic torque, i.e., the closed-loop system responds dynamically nonlinearly if $\epsilon \neq 1$. However, from the control law given by equation (18), notice that only $\epsilon=0$ will ensure a linear angular velocity feedback. Hence for the range

$$
0<\epsilon<1
$$

both the control law and the corresponding closed-loop response involve nonlinear dynamics. A consequence of this is that linear stability analysis and control design methods may yield unreliable results.

- This special form of the control law is analogous to previous results such as [3], [4], [5], and [6]. 
Consider stabilizing the vehicle attitude using only angular velocity feedback. Obviously without attitude feedback, the vehicle's attitude cannot be tracked to any commanded value but it can be regulated to some constant. Theorem 2 applies with the added condition of zero attitude feedback gains, $G_{\nu}=0_{3 \times 4}$. However, notice that the negative definite condition on the key angular velocity feedback gain parameter, $P$, does not provide a clear guidance for designing this gain matrix for a physical application. For this reason, we consider an additional condition on this gain matrix, namely, we limit the gain parameter, $P$, to a diagonal structure. The mathematical structure of the resulting feedback-linearized closed loop system is analogous to the damped open-loop system as studied in some detail in [2] for sizing the requirement on angular momentum bias. This analogy provides added insight for controller design.

Theorem 3 (Angular Velocity Feedback Only, $P=\operatorname{diag}\left(a_{1}, a_{2}, a_{3}\right)<0$ ) For the rotational equations of motion as given in (2), the feedback control torque

$$
\phi_{\tau}=\phi_{\text {gyro }}(\rho)+G_{\omega} \omega-\tau_{c}
$$

guarantee that the vehicle dynamics in (2) will be globally asymptotically stable $\left(\omega \rightarrow 0_{3 \times 1}\right.$ as $\left.t \rightarrow \infty\right)$ if the feedback gain matrix satisfies the following:

$$
\begin{aligned}
G_{\omega} & =\left[P-(1-\rho) \Delta_{\lambda}\right] J \\
P & =\operatorname{diag}\left(a_{1}, a_{2}, a_{3}\right), \quad a_{i}<0, \quad i=1,2,3 \\
J & =\operatorname{diag}\left(J_{1}, J_{2}, J_{3}\right) \\
\Delta_{\lambda} & =\delta_{\lambda}\left[\begin{array}{lll}
0 & 1 & 0 \\
1 & 0 & 0 \\
0 & 0 & 0
\end{array}\right]
\end{aligned}
$$

where

$$
\phi_{\text {gyro }}(\rho)=\omega^{\times} J \omega+\rho \omega^{\times} h_{B}
$$

and

$$
\rho \in \mathcal{R}: 0 \leq \rho \leq 1
$$

The $\phi_{\text {gyro }}(\rho)$ term in above control law fully feedback-linearizes the dynamics, resulting in the linear, timeinvariant closed loop system:

$$
\dot{\omega}=J^{-1} A J \omega
$$

where

$$
\begin{aligned}
A & =\left[\begin{array}{rrr}
a_{1} & -\gamma & 0 \\
\gamma & a_{2} & 0 \\
0 & 0 & a_{3}
\end{array}\right] \\
\gamma & =(1-\rho) \lambda \\
\lambda & =\frac{1}{2}\left(\lambda_{p_{1}}+\lambda_{p_{2}}\right)
\end{aligned}
$$

Since eigenvalues are invariant with respect to a similarity transformation, the eigenvalues of the closed loop system in equation (36) are:

$$
\begin{aligned}
\lambda_{1,2} & =-\zeta \omega_{n} \pm j \omega_{n} \sqrt{1-\zeta^{2}} \\
\lambda_{3} & =a_{3}
\end{aligned}
$$


where $\zeta$ and $\omega_{n}$ denote the damping ratio and natural frequency of the roll-pitch closed loop dynamics and are related to the design parameters by

$$
\begin{aligned}
\zeta \omega_{n} & =-\frac{1}{2}\left(a_{1}+a_{2}\right) \\
\omega_{n}^{2} & =\gamma^{2}
\end{aligned}
$$

As stated above, Theorems 2 and 3 are simplified restrictions of Theorem 1 that provide additional insight for control design: Theorem 2 for attitude tracking and trades on feedback linearization and gyroscopic torque compensation, and Theorm 3 for attitude stabilization. Focussing on the latter issue, note from equations $(39,42,43)$, that Theorem 3 gives three parameters that can be manipulated to adjust the natural frequency and damping ratio of the roll-pitch dynamics: $a_{1}, a_{2}$, and $\rho$. These parameters are selected by making two assumptions. First, assume that only the closed loop frequency response is of interest, rather than mode shapes. Secondly, invoke physical intuition to assume that the least amount of control effort will be expended if the control law is dedicated to providing damping, while leaving the closed loop frequency equal to its open loop value. The first assumption and equation (42) give

$$
\zeta \omega_{n}=-\bar{a} \quad a_{1}=a_{2}=\bar{a}
$$

The second assumption and equation (22) give

$$
\omega_{n}=\frac{h_{B}}{\sqrt{J_{1} J_{2}}}
$$

and from equation (43),

$$
\rho=1-\frac{2 \sqrt{J_{1} J_{2}}}{J_{1}+J_{2}}
$$

In summary, to obtain desirable closed loop transient properties, $\rho$ can be chosen from equation (46) to attain a natural frequency of $\omega_{n}$ while $\left(a_{1}, a_{2}\right)$ can be chosen to satisfy equation (42) for a selected damping ratio, $\zeta$. The simplest choice for these latter is to set them equal, per equation (44).

\section{Attitude Control via Classical Feedback}

In order to obtain simple heuristics for control the following reasonable assumptions are made

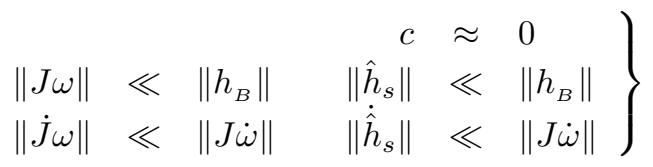

With the above assumptions, the following approximate equations are obtained:

$$
\begin{aligned}
\dot{v} & \approx-\omega^{\times} v+C_{b o} g_{o}+\frac{1}{m} \phi_{f} \\
J \dot{\omega} & \approx-\omega^{\times}\left(J \omega+h_{B}\right)+\phi_{\tau}
\end{aligned}
$$

These approximate equations are used only for the purpose of deriving control laws; all closed loop simulations shown in this study are based on the full equations.

$$
7 \text { of } 19
$$

American Institute of Aeronautics and Astronautics 
Consider an attitude control law based on classical feedback ideas. The approximate attitude dynamical equations, for an air vehicle which has a significant bias angular momentum, i.e., gyrically dominated in the body $z$-axis, as given by equations (49), are rewritten in the following expanded form:

$$
\begin{aligned}
& \dot{\omega}_{1} \approx-\lambda_{p_{1}} \omega_{2}+\frac{\tau_{1}}{J_{1}} \\
& \dot{\omega}_{2} \approx \lambda_{p_{2}} \omega_{1}+\frac{\tau_{2}}{J_{2}} \\
& \dot{\omega}_{3}=-\left(\frac{J_{2}-J_{1}}{J_{3}}\right) \omega_{1} \omega_{2}+\frac{\tau_{3}}{J_{3}}
\end{aligned}
$$

where

$$
\phi_{\tau} \triangleq\left(\tau_{1}, \tau_{2}, \tau_{3}\right)^{T}
$$

\section{A. Roll-Pitch damping by angular velocity feedback}

Consider the feedback control law:

$$
\begin{aligned}
& \frac{\tau_{1}}{J_{1}}=k_{D_{11}} \omega_{1}+k_{D_{12}} \omega_{2}+u_{1} \\
& \frac{\tau_{2}}{J_{2}}=k_{D_{21}} \omega_{1}+k_{D_{22}} \omega_{2}+u_{2}
\end{aligned}
$$

where $\left(u_{1}, u_{2}\right)$ are attitude error dependent feedback terms used subsequently to track commanded attitudes. To determine the gains, $\left(k_{D_{11}}, k_{D_{12}}, k_{D_{21}}, k_{D_{22}}\right)$, consider the closed-loop dynamical equations:

$$
\left\{\begin{array}{c}
\dot{\omega}_{1} \\
\dot{\omega}_{2}
\end{array}\right\} \approx\left[\begin{array}{cc}
k_{D_{11}} & -\lambda_{p_{1}}+k_{D_{12}} \\
\lambda_{p_{2}}+k_{D_{21}} & k_{D_{22}}
\end{array}\right]\left\{\begin{array}{l}
\omega_{1} \\
\omega_{2}
\end{array}\right\}+\left\{\begin{array}{l}
u_{1} \\
u_{2}
\end{array}\right\}
$$

The above closed-loop roll-pitch dynamics clearly correspond to the damped open-loop dynamics as given by equation (3.1) found in page 7 of [2]. In particular, $\left(k_{D_{11}}, k_{D_{22}}\right)$ correspond to damping coefficients, $\left(k_{D_{12}}, k_{D_{21}}\right)$ correspond to stiffness changes modifying the precession frequency, and $\left(u_{1}, u_{2}\right)$ correspond to external torque disturbances. To mitigate control requirements, we do not wish to change the precession frequency of the gyrating open-loop system so that we choose

$$
k_{D_{12}}=k_{D_{21}}=0
$$

Additionally, we ignore the "external torque disturbances" so that the roll-pitch closed-loop dynamical equations given in equation (56) reduces to the following:

$$
\left\{\begin{array}{c}
\dot{\omega}_{1} \\
\dot{\omega}_{2}
\end{array}\right\} \approx\left[\begin{array}{cc}
k_{D_{11}} & -\lambda_{p_{1}} \\
\lambda_{p_{2}} & k_{D_{22}}
\end{array}\right]\left\{\begin{array}{l}
\omega_{1} \\
\omega_{2}
\end{array}\right\}
$$

The closed-loop eigenvalues of the above system are given by (see equations (3.5) to (3.8) on page 7 of [2)]

$$
\lambda, \lambda^{*}=-\zeta \omega_{o} \pm j \omega_{o} \sqrt{1-\zeta^{2}}
$$

where

$$
\begin{aligned}
\zeta & =-\frac{1}{2 \omega_{o}}\left(k_{D_{11}}+k_{D_{22}}\right) \\
\omega_{o} & =\lambda_{o} \sqrt{1+\frac{k_{D_{11}} k_{D_{22}}}{\lambda_{o}^{2}}} \\
\lambda_{o} & =\frac{h_{B}}{\sqrt{J_{1} J_{2}}}
\end{aligned}
$$

$$
8 \text { of } 19
$$


At this point we can select a desired damping ratio and then solve for the gains, $k_{D_{11}}$ and $k_{D_{22}}$, from equations (60) and (61). However, as a simpler baseline approach, consider the following additional assumption:

$$
k_{D_{11}}=k_{D_{22}}=k
$$

With this simplifying assumption, equations $(60,61)$ give the following design condition:

$$
k=-\lambda_{o} \frac{\zeta}{\sqrt{1-\zeta^{2}}}<0
$$

In principle, one can choose an arbitrary closed-loop damping ratio without changing the precession frequency of the open-loop system by choosing the angular velocity feedback gains that satisfies equations $(60,61,57)$. Based on equation (64), a design rule of thumb could be inferred for the following nominal condition:

$$
-\frac{k}{\lambda_{o}} \approx \zeta ; \quad \zeta \leq 0.3
$$

which indicates that the ratio of the angular rate gains to the precession frequency is indicative of the vehicle damping ratio.

\section{B. Attitude tracking by attitude error feedback}

With the asymptotic convergence of $\omega_{1}$ and $\omega_{2}$ guaranteed by angular velocity feedback as described earlier, the requirement for convergence to commanded attitude angles still remains. This tracking is achieved by feeding back attitude errors, as outlined next.

Suppose

$$
\tau_{2}=0
$$

so that the pitch-axis dynamics from equation (51) become

$$
\dot{\omega}_{2} \approx \lambda_{p_{2}} \omega_{1}
$$

By taking the time derivative of pitch equation (67), substituting (50) for $\dot{\omega}_{1}$, and using the roll axis control law given by equation (54), and substituting for $\omega_{1}$ using equation (67), it can be shown that

$$
\ddot{\omega}_{2}-\frac{\lambda_{p_{2}}}{\lambda_{p_{1}}} k_{D_{11}} \dot{\omega}_{2}+\left(\lambda_{p_{2}} \lambda_{p_{1}}-\lambda_{p_{2}} k_{D_{12}}\right) \omega_{2} \approx \lambda_{p_{2}} u_{1}
$$

We make the following observations on equation (68):

- The pitch angular velocity state is uncoupled when viewed as a second-order oscillator.

- The pitch angular velocity is driven by roll torque.

- The coefficients of the second-order oscillator are such that any transient free response will be asymptotically stable.

Since the asymptotic convergence, $\omega_{2} \rightarrow 0$ as $t \rightarrow \infty$, does not ensure convergence to a commanded pitch angle, the additional control input torque component, $u_{1}$, is introduced. So assuming the small angle linear kinematics for pitch axis rotation,

$$
\dot{\theta}_{2}=\omega_{2}
$$

it can be shown that a PI feedback of the pitch angle error

$$
\begin{gathered}
u_{1}=-\left(k_{P_{1}}+\frac{1}{s} k_{I_{1}}\right)\left(\theta_{2}-\theta_{2}^{c m d}\right) \\
9 \text { of } 19
\end{gathered}
$$


will guarantee convergence to any step command in pitch angle, $\theta_{2}^{c m d}$. Notice that the pitch angle error is fed back as a commanded torque in the roll axis, which is intuitively consistent for a gyrically dominant system with the bias momentum in the yaw axis. However, design details for choosing gains for the PI control law are not addressed in this study given the availability of various techniques based on classical control design ideas (see for example Chapter 3 in [7]).

Using the above approach but assuming $\tau_{1}=0$, the required PI feedback to track commanded roll angle takes the form

$$
u_{2}=\left(k_{P_{2}}+\frac{1}{s} k_{I_{2}}\right)\left(\theta_{1}-\theta_{1}^{c m d}\right)
$$

The roll angle error is fed back as a commanded torque in the pitch axis. As for tracking yaw angles, the bias momentum which strongly cross couples roll and pitch axes do not affect the yaw dynamics except through the nonlinear vehicle gyroscopic component $\left(\frac{J_{2}-J_{1}}{J_{3}}\right) \omega_{1} \omega_{2}$, which is a small, compared to the wheel gyroscopic torques, $\lambda_{p_{1}} \omega_{2}$ and $\lambda_{p_{2}} \omega_{1}$. Hence tracking the yaw angles will involve a direct application of PI feedback of the yaw angle errors.

To summarize, the feedback control law for providing damping and attitude tracking is as follows:

$$
\begin{aligned}
\tau_{1}^{c m d} & =J_{1}\left[k_{D_{11}} \omega_{1}-\left(k_{P_{1}}+\frac{k_{I_{1}}}{s}\right)\left(\theta_{2}-\theta_{2}^{c m d}\right)\right] \\
\tau_{2}^{c m d} & =J_{2}\left[k_{D_{22}} \omega_{2}+\left(k_{P_{2}}+\frac{k_{I_{2}}}{s}\right)\left(\theta_{1}-\theta_{1}^{c m d}\right)\right] \\
\tau_{3}^{c m d} & =J_{3}\left[k_{D_{3}} \omega_{3}-\left(k_{P_{3}}+\frac{k_{I_{3}}}{s}\right)\left(\theta_{3}-\theta_{3}^{c m d}\right)\right]+\left(J_{2}-J_{1}\right) \omega_{1} \omega_{2}
\end{aligned}
$$

The yaw axis control law is composed of a feedback linearization term, a linear feedback of yaw angular velocity to provide damping and a PI feedback of the yaw angle error for tracking. The roll and pitch control laws consist of two components; a linear feedback of roll and pitch angular velocities to provide damping and PI feedback of the roll and pitch angle errors for tracking. However, the tracking angular errors are crosscoupled. The gyrically dominant influence of the bias angular momentum placed along the vehicle body yaw axis essentially feedback-linearizes the roll-pitch dynamics. Hence there is no need to use nonlinear feedback control for linearization. Finally, note that damping ratios on the precession frequency can be arbitrarily placed using for example, equation (64), while not changing the open loop precession frequency.

\section{Uncoupled small angle attitude kinematics}

With this single-loop approach, the angular tracking error terms are treated independently and conveniently so that no rotation sequence need to be considered. On the other hand, the kinematic nonlinearity inherent for large angular motion (say beyond 30 degrees deviation) limits the applicability of this control law. However, notice that the angular rate and acceleration feedback terms still apply for large angular motion so that a judicious choice of control gains may result in satisfactory closed loop performance. For small angular motions, these attitude tracking errors can be approximated directly from the Euler parameters as follows (see for example Chapter 2 in [8]):

$$
\begin{aligned}
& \theta_{2}=\arcsin \left[-C_{b o_{13}}(\nu)\right] \approx 2 \nu_{2} \\
& \theta_{3}=\arcsin \left[C_{b o_{12}}(\nu) / \cos \theta_{2}\right] \approx 2 \nu_{1} \\
& \theta_{1}=\arcsin \left[C_{b o_{23}}(\nu) / \cos \theta_{2}\right] \approx 2 \nu_{3}
\end{aligned}
$$

Notice that the above equations relate 321-sequence Euler angles (in radians) to the unique direction cosine matrix, which is parameterized by a set of Euler parameters. As is well known, the above relationship suffers a kinematic singularity when the pitch angle equals $\pm \pi / 2$. 


\section{Control Law Comparison via Simulation}

This section describes numerical simulation experiements conducted to characterize the performance of the linear and nonlinear (Lyapunov) controllers for a range of magnitudes of the angular momentum bias $h_{B}$. As mentioned in the Introduction, it is desirable to employ the smallest $h_{B}$ that will adequately stiffen the vehicle's attitude dynamics, since that results in the smallest flywheel mass. In this study, the tradeoff between vehicle mass and closed-loop performance is realized by posing several flywheels with differing mass but common physical assumptions: All flywheels were assumed to be thin steel rings with tensile strength of $80 \mathrm{kpsi}$ and a $30 \mathrm{krpm}$ rotational rate, with their radii chosen so that the hoop stress would be 80kpsi. Four cases were considered, indexed on the flywheel's fraction of the total vehicle mass. These were $m_{\text {flywheel }}=\{0,1 \%, 5 \%, 20 \%\} \cdot m_{\text {vehicle }}$. For each of these four cases, a classical controller and a nonlinear controller were formulated, and the vehicle was controlled to follow a commanded trajectory in the face of aerodynamic disturbances.

Figure 1 shows a sketch of the simulated vehicle used for the control comparison experiments, along with its key nominal parameters. It is a small square platform with a shrouded propeller at each corner. The

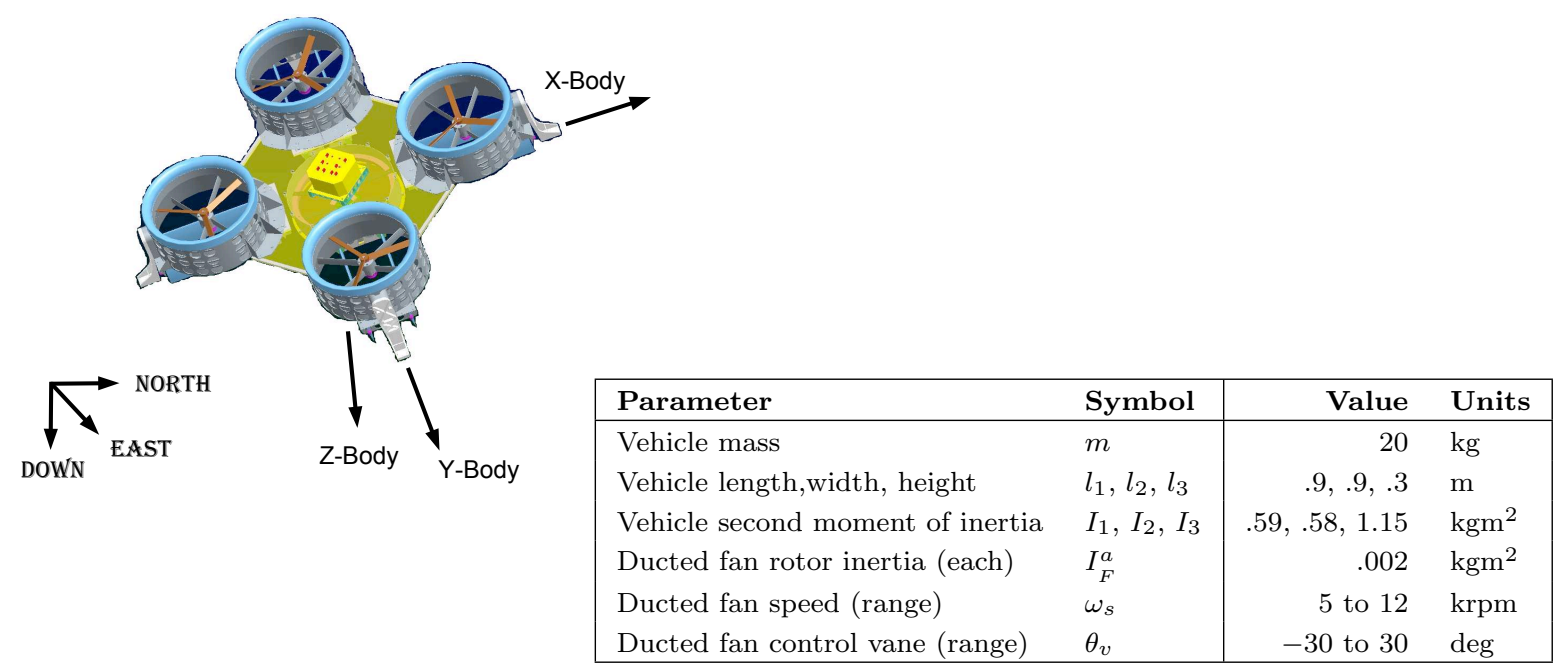

Figure 1. Schematic of a generic quad ducted fan with bias momentum wheel.

vehicle's body axes have their origin at the center of mass, and are oriented as shown more clearly in Figure 2 , with the $z$-body axis pointed into the page. Thrust vectoring vanes are mounted downstream of each propeller, and each set of vanes diverts the thrust along the body axis normal to the body axis that passes through the thruster. Figure 2 displays both the vehicle body axes and four local coordinate systems for the four thrusters: $F_{F_{j}}, j=1,2,3,4$. The thrust deflections due to thrust vectoring vanes are parallel to the $y$ axes of the $F_{F_{j}}$ frames. The momentum wheel unit is assumed to be rigidly attached to the center of the vehicle which, itself, is assumed rigid. The spin axis is assumed to be collinear with the vehicle yaw axis.

The commanded trajectory to be followed by the vehicle is shown in Figure 3, and consists of a vertical ascent that transitions to level cruise. Starting from a hovering position, the vehicle is commanded to climb at a flight path angle of 80 degrees while accelerating for a duration of 10 seconds. This is followed by a transition of flight path angle from 80 degrees to 0 degrees in 5 seconds while continuing to accelerate. At the end of 15 seconds the vehicle is commanded to a level flight while the airspeed continues to accelerate for the next 5 seconds. From 20 seconds into the flight, steady level cruise is commanded for the next 20 seconds. Note that for most of this trajectory, the commanded attitude is constant. 


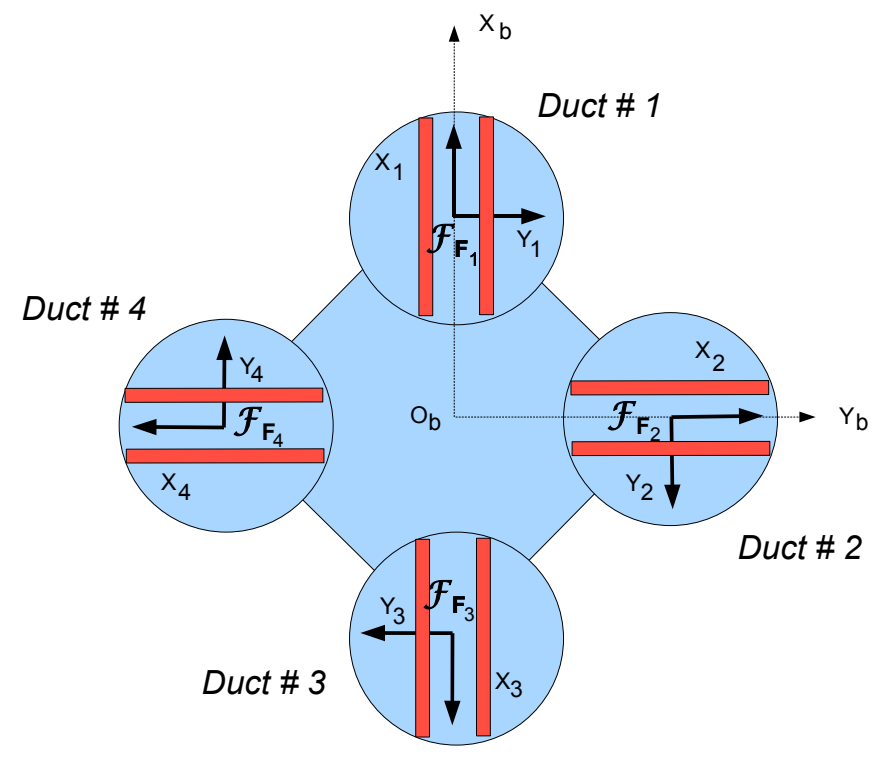

Figure 2. Top view of ducted fan frame orientations relative to platform body frame.

The air disturbances to which the vehicle is subjected during flight were assumed to come from three sources: (1) basic aerodynamic loads as driven by the steady wind states $\left(V_{T}, \alpha, \beta, \omega\right),(2)$ air turbulence as defined by a Dryden model as described for example in Chapter 4.5 of [9], and (3) ground effects. The basic aerodynamic loads on the vehicle are shown in the left side of Figure 4. Notice the steep increase in drag forces and the pitch down aerodynamic torque as the vehicle increases to cruise speeds. The net aerodynamic loads with the additional simulated turbulence as described by a Dryden model with the following turbulence parameters: intensity $\sigma_{w}=3.8 \mathrm{~m} / \mathrm{s}$, scales $\left(L_{x}, L_{y}, L_{z}\right)=(121,60,30) \mathrm{m}$, are shown in the right side of Figure 4. To simulate the air loads on a vehicle that takes off vertically from a ground position and then cruising at low altitudes which may be gusty due to ground obstacles such as buildings and uneven landscape, random gust torques having a bandwidth of $3 \mathrm{Hertz}$ in all axes are assumed. The amplitude of this random signal is

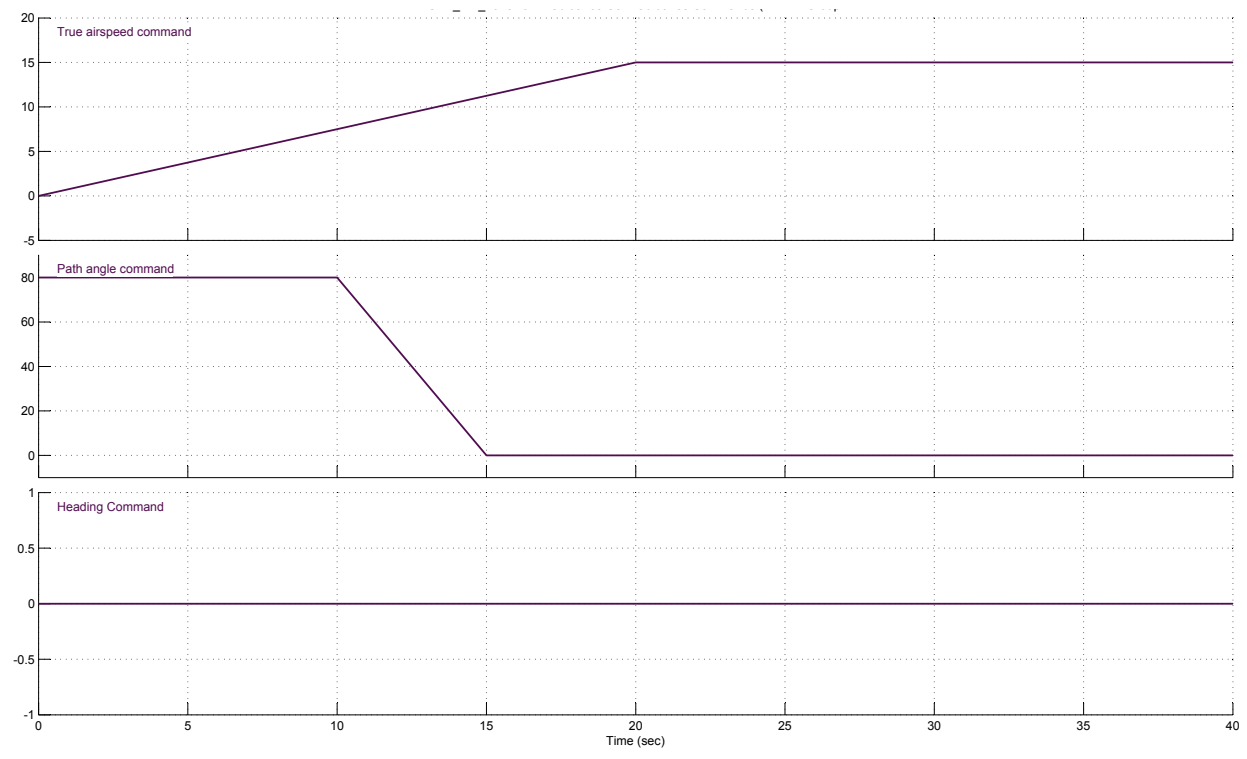

Figure 3. Commanded vehicle trajectory: airspeed (m/s), path angle (deg), heading (deg). 

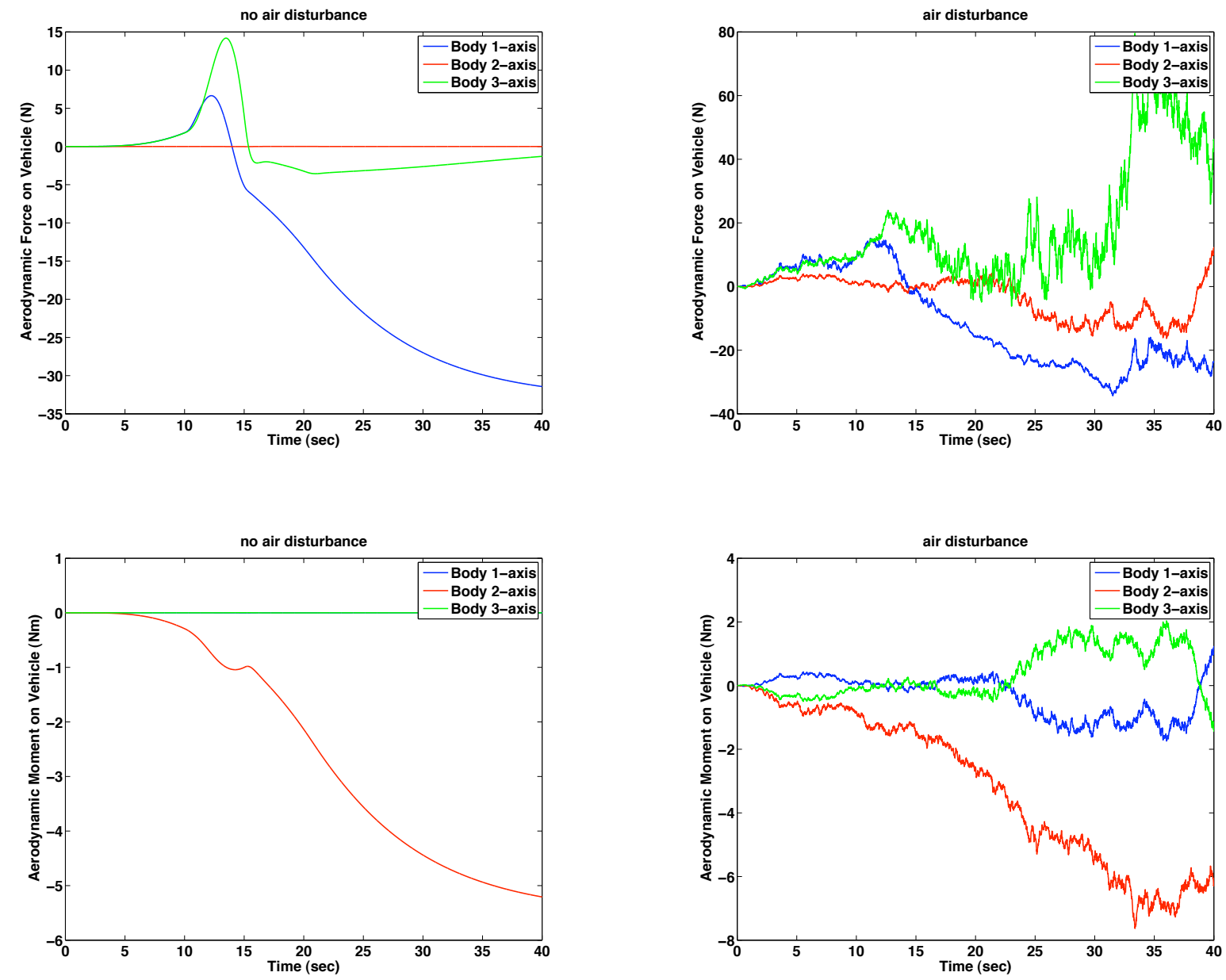

Figure 4. Aerodynamic Loads on the Vehicle About Trim: without turbulence (left), with turbulence (right). 
set to taper off linearly from ground to an altitude of 5 meters where it is set to zero above it. In addition, low level random disturbance torques are added to account for persistent gusts in low altitude atmosphere. Figure 5 shows the simulated random gust air loads due to the ground effects and low altitude flight.

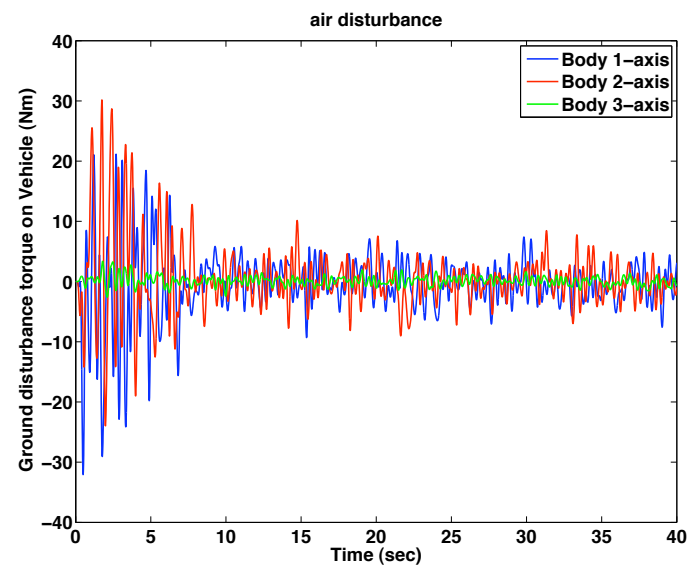

Figure 5. Random gust loads on the vehicle due to ground effects and low altitude flight.

Figures 6 and 7 summarize the closed-loop performance of the vehicle in following the control trajectory in terms of rms values; for Figure 6, the deviation of attitude and velocities from commanded values, and for Figure 7, deviations of control variables from their trim settings. The rms values are computed as the square root of the time integral of the squared deviation, divided by the integration interval. The "case numbers" labelled on the horizontal axes of the plots refer to the combination of control law type and flywheel (bias momentum) size. The prefix "C" refers to a classical control design and "L" to a nonlinear one. The numbers $0,1,2,3$, refer to $0 \%, 1 \%, 5 \%$, and $20 \%$ flywheel mass fraction, respectively.

The first thing to note in Figure 6 is that all the bars for case $\mathrm{C} 1$ extend to the top of the charts. This is because the authors were unsuccessful in finding a stabilizing linear controller with the structure described in Section 3. The $1 \%$ flywheel for this case did not generate enough $h_{B}$ to gyrically dominate the dynamics, but did generate enough to invalidate the linear model assumed by the controller. The case $\mathrm{C} 0$, with no bias momentum, actually worked better. In all cases, the "L" controllers did somewhat better in terms of rms behavior than the corresponding "C"s, particularly in the case of L1 versus $\mathrm{C} 1$. The second thing to note in Figure 6 is that the performance of the linear and nonlinear control laws is quite comparable for the larger flywheel masses, though the Lyapunov controller was marginally better. In Figure 7 note that the Lyapunov controller appears to be somewhat more aggressive than the classical controller in its use of fan speed, though the controllers are otherwise comparable, at least at the $5 \%$ and $20 \%$ flywheel mass fractions. 

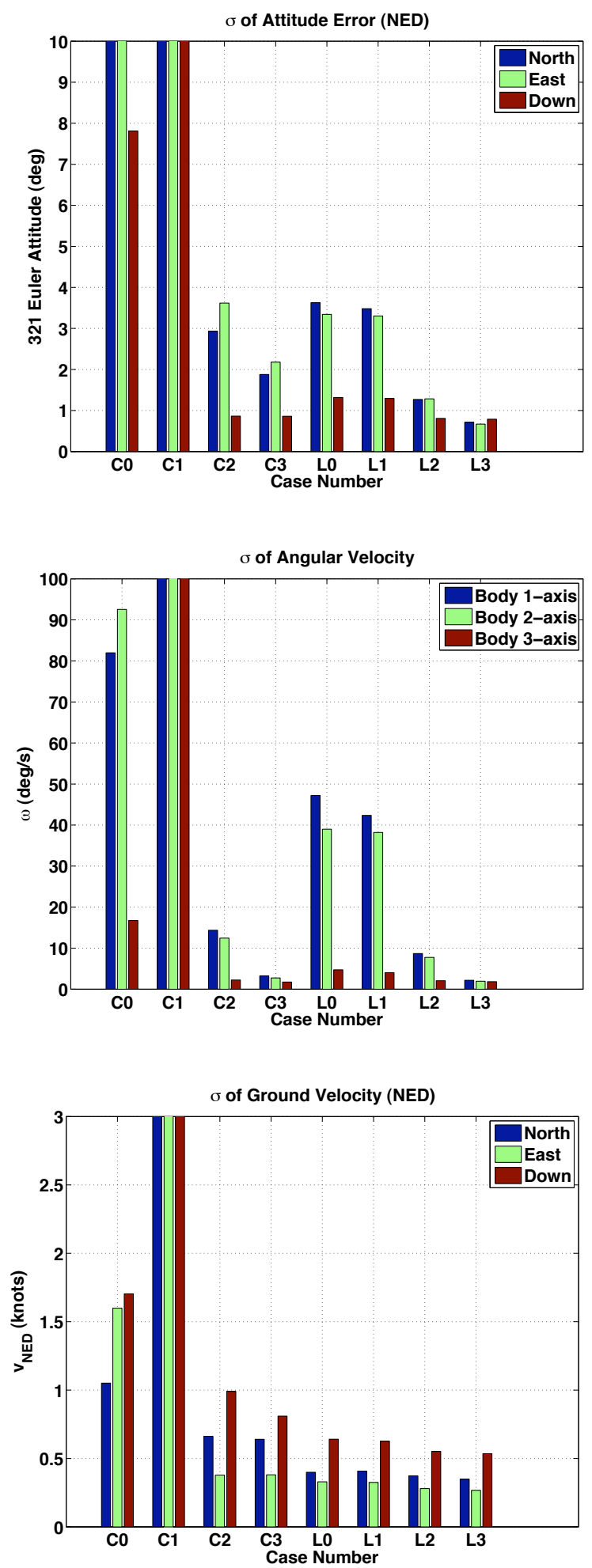

Figure 6. vehicle response comparisons. 

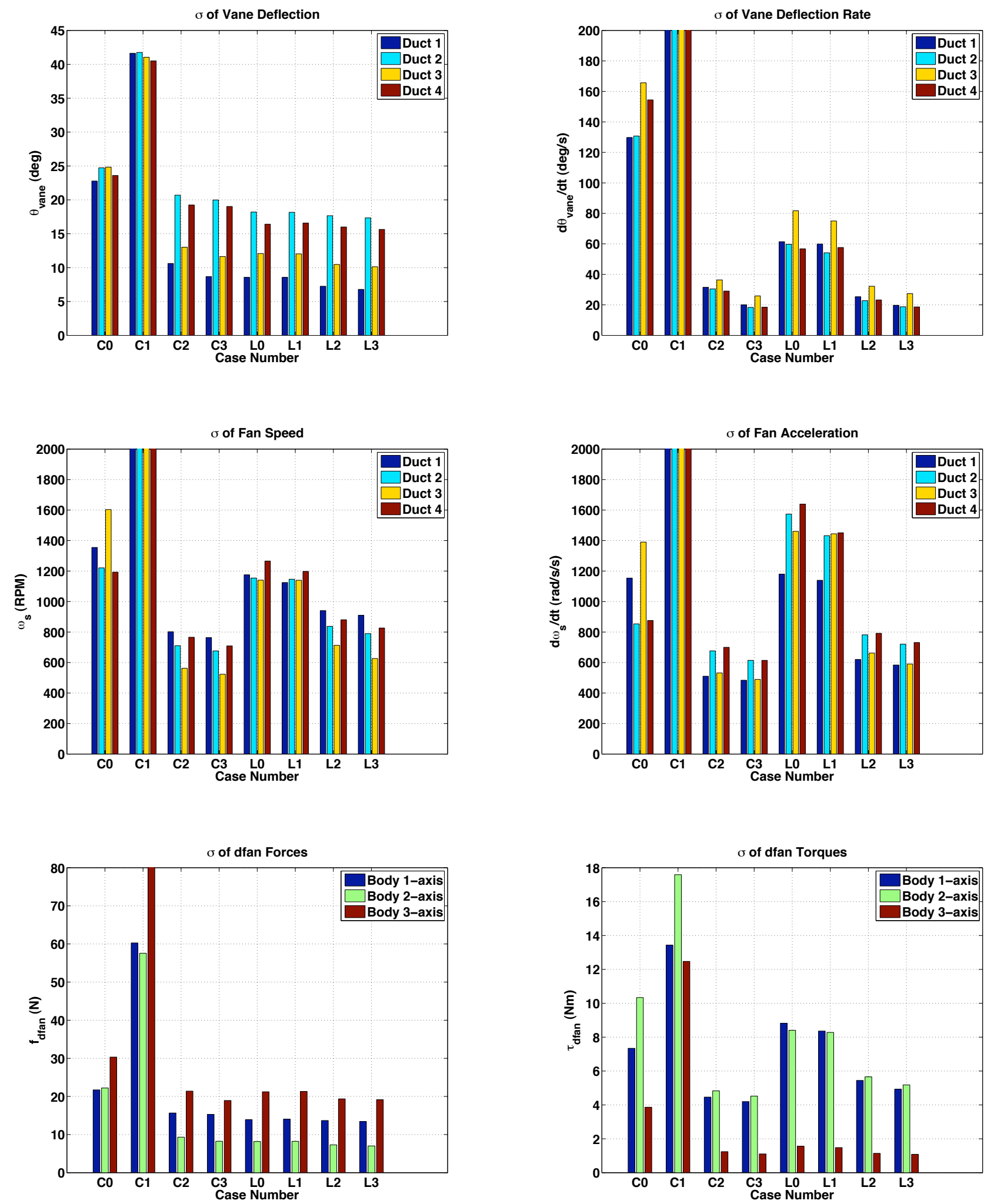

Figure 7. control effort comparisons. 


\section{Conclusions}

This paper has described two controller structures suitable for an atmospheric flight vehicle that includes a steady yaw-axis angular momentum bias. A nonlinear controller based on Lyapunov stability theory was described and its stability was proven, for the full formulation, and for two restricted cases that allowed a simplified gain selection approach. In addition to this, a classical PID-type linear controller structure was described for the case where the vehicle's attitude dynamics was gyrically dominant - where the angular momentum bias was sufficiently large that the nonlinearities in the dynamics were masked by the bias. It was shown that this controller structure requires that the PI roll and pitch error feedback must be crossfed. Both controllers were demonstrated by applying them to a simulation of a thrust-levitated vehicle that is commanded through a maneuver in which vertical flight transitions to horizontal in the presence of aero disturbances. The controllers were simulated for several levels of angular momentum bias, and it was seen that, while both controllers could provide good response for substantial angular momentum biasses, the nonlinear controller was necessary for controlling the system for a very small angular momentum bias. This was important because, in the quest for the lightest possible vehicle, there is considerable pressure to minimize the mass of the bias momentum flywheel.

\section{A. Proof of Theorems}

The proof is based on Lyapunov stability theory (for more details see for example [10]) and shows the asymptotic stability of the equilibrium point, $\left(\delta \omega=0_{3 \times 1}, \delta \nu=0_{4 \times 1}\right)$. The theorem assumes the dynamical system given by equations (2) whose attitude error kinematics are described by equation (9). The closed loop dynamical equations can be obtained by substituting the feedback control torque in equation (11) into the dynamical equations (2). However, to simultaneously prove Theorems 1 and 2, let us assume the more general form of $\phi_{\text {gyro }}$ as given in equation (15). This results in the closed loop response equations

$$
J \delta \dot{\omega}=-(1-\epsilon) \omega^{\times} J \omega-(1-\rho) \omega^{\times} h_{B}+G_{\omega} \delta \omega+G_{\nu} \delta \nu
$$

where $G_{\nu}$ is given by equation (12) and $G_{\omega}$ by equation (13).

For the above closed loop system, consider the following function about the null equilibrium point:

$$
V(\delta h, \delta \nu)=\frac{1}{2}\left(\begin{array}{c}
\delta h \\
\delta \nu
\end{array}\right)^{T}\left[\begin{array}{ll}
I_{3 \times 3} & \\
& k_{\nu} I_{4 \times 4}
\end{array}\right]\left(\begin{array}{c}
\delta h \\
\delta \nu
\end{array}\right)
$$

where

$$
\delta h \triangleq J \delta \omega
$$

The above choice of function satisfies the necessary Lyapunov function properties:

$$
V(0,0)=0, \quad \text { and } \quad V>0, \forall\left(\begin{array}{c}
\delta h \\
\delta \nu
\end{array}\right) \neq 0
$$

Notice that the variable $\delta h$ in the previous equations can be substituted by $\delta \omega$ without any change in the property of $V$ since $J$ is nonsingular.

It can be shown that along any solution trajectory of the system defined by equations (78) and (9),

$$
\begin{aligned}
\dot{V}= & \delta h^{T}\left[-(1-\epsilon) \omega^{\times} J \omega-(1-\rho) \omega^{\times} h_{B}+G_{\omega} \delta \omega+G_{\nu} \delta \nu\right] \\
& +\frac{1}{2} k_{\nu} \delta \nu^{T}\left[[\bar{\nu}]\left(\begin{array}{c}
0 \\
\delta \omega
\end{array}\right)+[\omega] \delta \nu\right]
\end{aligned}
$$


By partitioning and defining as given in equation (9) we can rewrite

$$
[\bar{\nu}]\left(\begin{array}{c}
0 \\
\delta \omega
\end{array}\right)=\bar{B} \delta \omega
$$

In addition, since $[\omega]$ is skew-symmetric

$$
\delta \nu^{T}[\omega] \delta \nu=0
$$

and furthermore, since the attitude feedback gains satisfy equation (12), the terms

$$
\delta h^{T} G_{\nu} \delta \nu+\frac{1}{2} k_{\nu} \delta \nu^{T} \bar{B} \delta \omega=0
$$

Hence, equation (82) 21) and $\lambda$ is defined by equation (39), to rewrite the $\dot{V}$ expression in equation (??) as follows:

$$
\dot{V}=-\delta h^{T}\left[(1-\epsilon) \omega-(1-\rho)\left(\begin{array}{c}
0 \\
0 \\
\lambda
\end{array}\right)\right]^{\times} J \omega+(1-\rho) \delta h^{T} \Delta_{\lambda} J \omega+\delta h^{T} G_{\omega} J^{-1} \delta h
$$

At this point, the proof for Theorems 1 and 2 are considered separately.

Theorem 1: $\quad \epsilon=1$ and $\rho=1$, i.e., the complete feedback linearization of all gyroscopic torques is assumed. The $\dot{V}$ expression in equation (86) reduces to

$$
\dot{V}=\delta h^{T} G_{\omega} J^{-1} \delta h
$$

To satisfy Lyapunov stability condition we note that in addition to conditions in equation (81):

$$
\begin{array}{ll} 
& \dot{V}=\delta h^{T} G_{\omega} J^{-1} \delta h \leq 0, \quad \forall(\delta h, \delta \nu) \\
\Leftarrow \quad & \dot{V}<0, \quad \forall \delta h \neq 0 \\
\Leftrightarrow \quad & G_{\omega} J^{-1}<0 \\
\Leftrightarrow \quad & \exists G_{\omega}: G_{\omega}=P J, \quad P \in \mathcal{R}^{3 \times 3}, \quad P<0
\end{array}
$$

The condition for the gain matrix, $G_{\omega}$, in equation (91) is identical to that of equation (13) found in Theorem 1. This proves the Lyapunov stability implicit in Theorem 1. To show that the system is actually asymptotically stable, note from equation (88) that

$$
\dot{V}=\delta h^{T} G_{\omega} J^{-1} \delta h=0 \quad \text { iff } \quad \delta h=0
$$

This suggests a proof of asymptotic stability by showing that the only feasible solution in the subset of solutions $(\delta h=0, \delta \nu)$ satisfying $\dot{V}=0$ is the null solution, which is our equilibrium solution of interest. By substituting these additional conditions into the closed loop system equation (78) and noting the requirement $k_{\nu}>0$, it follows that

$$
\bar{B}^{T} \delta \nu=0_{3 \times 1}
$$

It follows from equation (9) that a basis vector for the null space of $\bar{B}^{T}$ in the above equation is $\bar{\nu} \in \mathcal{R}^{4 \times 1}$. This means that any solution, $\delta \nu$ to the above equation is a scaling of $\bar{\nu}$ itself, and since an Euler parameter vector is unit norm bounded, we can conclude that $\delta \nu=0_{4 \times 1}$. Hence the only solution that satisfies $\dot{V}=0$ condition is the null solution $\left(\delta \omega=0_{3 \times 1}, \delta \nu=0_{4 \times 1}\right)$. By a corollary of the Lyapunov theorem (see for example, [10]), this proves asymptotic stability. Finally, observe that with the above choice of the Lyapunov function which is continuously differentiable, radially unbounded, and positive definite, the asymptotic stability result applies globally as well. 
Theorem 2: $\bar{\omega}=0$ but for any values of $\epsilon \in[0: 1]$ and $\rho \in[0: 1]$. This implies that

$$
\delta h=J \delta \omega=J \omega
$$

which represents the set of control problems involving attitude regulation. Using equation 94, the $\dot{V}$ expression in equation 86 reduces to

$$
\dot{V}=\delta h^{T}\left[(1-\rho) \Delta_{\lambda}+G_{\omega} J^{-1}\right] \delta h
$$

since

$$
\delta h^{T}\left[(1-\epsilon) \omega-(1-\rho)\left(\begin{array}{c}
0 \\
0 \\
\lambda
\end{array}\right)\right]^{\times} \delta h=0
$$

To satisfy Lyapunov stability condition note that:

$$
\begin{array}{ll} 
& \dot{V}=\delta h^{T}\left[(1-\rho) \Delta_{\lambda}+G_{\omega} J^{-1}\right] \delta h \leq 0, \quad \forall(h, \delta \nu) \\
\Leftarrow \quad & \dot{V}<0, \quad \forall \delta h \neq 0 \\
\Leftrightarrow \quad & (1-\rho) \Delta_{\lambda}+G_{\omega} J^{-1}<0 \\
\Leftrightarrow & \exists G_{\omega}: G_{\omega}=\left[P-(1-\rho) \Delta_{\lambda}\right] J, \quad P \in \mathcal{R}^{3 \times 3}, \quad P<0
\end{array}
$$

The condition for the gain matrix, $G_{\omega}$, in equation (100) is identical to that of equation (20) in Theorem 2. This proves the Lyapunov stability implicit in the Theorem. To show that the system is actually asymptotically stable, note from equation (97) that

$$
\dot{V}=\delta h^{T}\left[(1-\rho) \Delta_{\lambda}+G_{\omega} J^{-1}\right] \delta h=0 \quad \text { iff } \quad \delta h=0
$$

As in the asymptotic stability proof in Theorem 1, this suggests a proof based on showing that the only feasible solution in the subset of solutions $(\delta h=0, \delta \nu)$ satisfying $\dot{V}=0$ is the null solution. Hence the remaining steps involve substituting the particular additional conditions in Theorem 2 into the closed loop system equation (78). The end result is equation (93) with the conclusion that the only solution that satisfies $\dot{V}=0$ condition is the null solution. The reasons for global asymptotic stability holds here as well.

TheOREM 3: Theorem 3 is a special case of Theorem 2.

\section{References}

\footnotetext{
${ }^{1}$ Lim, K.B., and Moerder, D.D., "A new approach to attitude stability and control for low airspeed vehicles," AIAA Guidance, Navigation, \& Control Conference, August 16-19, 2004, Providence, RI, AIAA Paper 2004-5008.

${ }^{2}$ Lim, K.B., Shin, J.Y., and Moerder, D.M., "Bias Momentum Sizing for Hovering Dual-Spin Platforms", NASA TP 2006-214317, NASA Langley Research Center, May 2006. 1968.

${ }^{3}$ Mortensen, R.E., "A Globally stable linear attitude regulator", International Journal of Control, Vol. 8, pp. 297-302,

${ }^{4}$ Wie, B., and Barba, P.M., "Quarternion feedback for spacecraft large andle maneuvers", Journal of Guidance, Control, and Dynamics, Vol. 8, No. 3, pp. 360-365, 1985.

${ }^{5}$ Joshi, S.M., Kelkar, A.G., and Wen, J.T-Y., "Robust attitude stabilization of spacecraft using nonlinear quarternion feedback", IEEE Transactions on Automatic Control, Vol. 40, No. 10, pp. 1800-1803. 1995.

${ }^{6}$ Tsiotras, P., "New control laws for the attitude stabilization of rigid bodies," IFAC Symposium on Automatic Control in Aerospace, Palo Alto, CA, Sept. 12-16, 1994, pp. 316-321.

${ }^{7}$ Franklin, G.F., Powell, J.D., and Emami-Naeini, A., Feedback Control of Dynamic Systems, Addison-Wesley Publishing Co., Reading, Massachusetts, 1986.

${ }^{8}$ Hughes, P.C., Spacecraft Attitude Dynamics, John Wiley \& Sons, Inc., New York, 1986.

${ }^{9}$ Hoblit, F. M., Gust Loads on Aircraft: Concepts and Applications, AIAA Education Series, AIAA, Washington, DC, 1988.

${ }^{10}$ Khalil, H. K., Nonlinear Systems, Third Edition, Prentice Hall, Upper Saddle River, New Jersey, 2002. Chapter 4.
} 